\title{
NICELY SEMIRAMIFIED DIVISION ALGEBRAS OVER HENSELIAN FIELDS
}

\author{
KARIM MOUNIRH
}

Received 5 July 2004

This paper deals with the structure of nicely semiramified valued division algebras. We prove that any defectless finite-dimensional central division algebra over a Henselian field $E$ with an inertial maximal subfield and a totally ramified maximal subfield (not necessarily of radical type) (resp., split by inertial and totally ramified field extensions of $E$ ) is nicely semiramified.

\section{Introduction}

We recall that a nicely semiramified division algebra is defined to be a defectless finitedimensional valued central division algebra $D$ over a field $E$ with inertial and totally ramified radical-type (TRRT) maximal subfields [7, Definition, page 149]. Equivalent statements to this definition were given in [7, Theorem 4.4] when the field $E$ is Henselian. These division algebras, as claimed in [7, page 128], appeared in [10] as examples of division algebras with nonzero $S K_{1}$. The main purpose of this paper is to prove that over a Henselian field $E$, any central division algebra with inertial and totally ramified maximal subfields (resp., split by inertial and totally ramified field extensions of $E$ ) is nicely semiramified.

We precise that all rings considered in this work are assumed to be associative with a unit and all free modules are assumed to be finite-dimensional. A valued division algebra $D$ over a field $E$-we adopt the same valuative definitions as in [7] —is called defectless (over $E)$ if $[D: E]=[\bar{D}: \bar{E}]\left(\Gamma_{D}: \Gamma_{E}\right)$, where $[\bar{D}: \bar{E}]\left(\right.$ resp., $\left.\left(\Gamma_{D}: \Gamma_{E}\right)\right)$ is the residue degree (resp., ramification index) of $D$ over $E$.

We recall that for any valued central division algebra $D$ over a field $E$, the center $Z(\bar{D})$ of $\bar{D}$ is a normal field extension of $\bar{E}$ and the mapping

$$
\begin{gathered}
\theta_{D}: \Gamma_{D} / \Gamma_{E} \longrightarrow \operatorname{Gal}(Z(\bar{D}) / \bar{E}), \\
\gamma+\Gamma_{E} \longmapsto \theta_{D}\left(\gamma+\Gamma_{E}\right): \bar{a} \longmapsto \overline{d a d^{-1}},
\end{gathered}
$$

where $d$ is an arbitrary element of $D$ such that $v(d)=\gamma(v$ being the valuation of $D)$, is a surjective group homomorphism [7, Proposition 1.7]. We say that $D$ is tame (over E) if 
it is defectless over $E, Z(\bar{D})$ is separable (hence Galois) over $\bar{E}$, and the characteristic of $\bar{E}$ does not divide the cardinal of the kernel of $\theta_{D}$. A subfield $K(\supseteq E)$ of $D$ is called inertial over $E$ if $[K: E]=[\bar{K}: \bar{E}]$ and $\bar{K}$ is separable over $\bar{E}$.

Now, let $F$ be an associative ring with a unit, and $\Gamma$ a totally ordered abelian group. We say that $F$ is a graded ring of type $\Gamma$ if there are subgroups $F_{\gamma}(\gamma \in \Gamma)$ of $F$ such that $F=\bigoplus_{\gamma \in \Gamma} F_{\gamma}$ and $F_{\gamma} F_{\delta} \subseteq F_{\gamma+\delta}$, for all $\gamma, \delta \in \Gamma$. In this case, the set $\Gamma_{F}=\left\{\gamma \in \Gamma \mid F_{\gamma} \neq 0\right\}$ is called the support of $F$. If $x \in F_{\gamma}$ for some $\gamma \in \Gamma_{F}$, we say that $x$ is a homogeneous element of $F$. In particular, if $x$ is a nonzero element of $F_{\gamma}$, we say that $x$ has grade $\gamma$ and we write $\operatorname{gr}(x)=\gamma$. We denote by $F^{h}$ (resp., $F^{*}$ ) the set of homogeneous (resp., nonzero homogeneous) elements of $F$. A graded ring $F$ which is commutative and for which all nonzero homogeneous elements are invertible is called a graded field.

Let $F$ be a commutative graded ring of type $\Gamma$. A (left) $F$-module (resp., $F$-algebra) $A$ is called a graded $F$-module (resp., a graded $F$-algebra) (of type $\Gamma$ ) if $A=\bigoplus_{\delta \in \Gamma} A_{\delta}$ and $F_{\gamma} A_{\delta} \subseteq A_{\gamma+\delta}$, for all $\gamma, \delta \in \Gamma$ (resp., if $A$ is a graded ring of type $\Gamma$ and $F_{\gamma} \subseteq A_{\gamma}$, for all $\gamma \in \Gamma$ ). In particular, if $F$ is a graded field, then graded $F$-algebras (resp., commutative graded $F$-algebras) for which homogeneous elements are invertible are called graded division $F$-algebras (resp., graded field extensions of $F$ ).

A graded $F$-module (resp., a graded $F$-algebra) is also called a graded module (resp., a graded algebra) over $F$.

Let $F$ be a graded field of type $\Gamma$. Since $\Gamma$ is totally ordered, then any graded division algebra $A$ over $F$ is a domain. We may then consider the algebra of central quotients of $A$ that we denote by $\mathrm{Cq}(A)$. Remark that if $A$ is a graded field extension of $F$, then $\mathrm{Cq}(A)$ coincides with the fraction field $\operatorname{Frac}(A)$ of $A$.

One can easily see that if $F$ is a graded field, then any graded $F$-module $M$ is free over $F$. Indeed, by [1, Theorem 3, page 29] any maximal E-linearly independent subset of homogeneous elements of $M$ is a basis of $M$ over $E$.

A graded field extension $K$ of a graded field $F$ is called totally ramified over $F$ if $[K$ : $F]=\left(\Gamma_{K}: \Gamma_{F}\right)$. It is called unramified over $F$ if $[K: F]=\left[K_{0}: F_{0}\right]$ and $K_{0}$ is separable over $F_{0}$. Finally, $K$ is called tame over $F$ if $K_{0}$ is separable over $F_{0}$ and $\Gamma_{K} / \Gamma_{F}$ has no $p$-torsion, where $p=\operatorname{char}(F)$.

If $F$ is the center of a graded division algebra $D$, then $D$ is called a graded central division algebra (GCDA) over $F$. We recall that in the same way as for (ungraded) fields, we can define the graded Brauer group $\operatorname{GBr}(F)$ of $F$, where GCDAs play the same role as central division algebras (CDAs) over (ungraded) fields (see [2, Section 5] or [6, Section 3]).

Let $F$ be a graded field of type $\Gamma$ and $D$ a GCDA over $F$. Then a natural valuation $v$ can be defined on $\mathrm{Cq}(D)$ by setting $v(d)=\operatorname{gr}(d)$ for any $d \in D^{*}$ (see $[3$, Section 4] or [6, Section 4]). Throughout the rest of the paper, such $v$ will be called the canonical valuation of $\mathrm{Cq}(D)$. We recall that $\mathrm{Cq}(D)$, with respect to $v$, is a tame $\operatorname{CDA}$ over $\operatorname{Frac}(F)$ [3, Proposition 4.1(3)].

Conversely, for any defectless valued division algebra $D$ with valuation $v$ over a field $E$, we define $E^{\gamma}=\{x \in E \mid v(x) \geq \gamma\}, E^{>\gamma}=\{x \in E \mid v(x)>\gamma\}, D^{\gamma}=\{x \in D \mid v(x) \geq \gamma\}$, and $D^{>\gamma}=\{x \in D \mid v(x)>\gamma\}$. Obviously, $E^{>\gamma}$ (resp., $D^{>\gamma}$ ) is a subgroup of the additive group $E^{\gamma}$ (resp., $D^{\gamma}$ ). So, we can define the quotient groups $G E_{\gamma}=E^{\gamma} / E^{>\gamma}$ and $G D_{\gamma}=D^{\gamma} / D^{>\gamma}$. For $x \in E \backslash\{0\}$ (resp., $x \in D \backslash\{0\}$ ), we denote by $\tilde{x}$ the element $x+E^{>v(x)}$ of $G E_{v(x)}$ 
(resp., $x+D^{>v(x)}$ of $G D_{v(x)}$ ). One can easily check that the additive group $G E=\bigoplus_{\gamma \in \Gamma} G E_{\gamma}$ endowed with the multiplication law defined by $\tilde{x} \tilde{y}=\tilde{x y}$ is a graded field. Analogously, $G D=\bigoplus_{\gamma \in \Gamma} G D_{\gamma}$ is a graded division algebra over $G E$ [2, page 4281]. We recall that $D$ is tame over $E$ if and only if GD is a GCDA over GE [2, Corollary 4.4(3)] (remark that since $D$ is defectless over $E$, then $[D: E]=[G D: G E])$.

Now, let $F$ be a graded field and let $D$ be a GCDA over $F$, and denote by $H \operatorname{Frac}(F)$ the Henselization of $\operatorname{Frac}(F)$ with respect to the canonical valuation of $\operatorname{Frac}(F)$ [4, Section 16], and let $H \mathrm{Cq}(D)=\mathrm{Cq}(D) \otimes_{\operatorname{Frac}(F)} H \operatorname{Frac}(F)$. Then, $D$ is graded isomorphic to $G H \mathrm{Cq}(D)$ by means of the mapping $x \mapsto \tilde{x}$, where $x$ is an arbitrary homogeneous element of $D$. Indeed, we have $D_{0}=(G H \mathrm{Cq}(D))_{0}$ and $\Gamma_{D}=\Gamma_{G H C q(D)}$. We write $D \cong_{g} G H C q(D)$. We also have $F \cong_{g} G H \operatorname{Frac}(F)$.

We recall that if $E$ is Henselian, then the tame part $\operatorname{TBr}(E)$ of the Brauer group $\operatorname{Br}(E)$ of $E$ (i.e., $\operatorname{TBr}(E)=\{[D] \in \operatorname{Br}(E) \mid D$ is a tame CDA over $E\}$ ) is isomorphic to $\operatorname{GBr}(G E)$ [6, Theorem 5.3]. Also, for any graded field $F, \operatorname{GBr}(F)$ is isomorphic to $\operatorname{TBr}(H \operatorname{Frac}(F))$ [6, Theorem 5.1]. These isomorphisms are index-preserving. We call them the canonical isomorphisms.

\section{Nicely semiramified division algebras over Henselian fields}

Let $F$ be a graded field and $K$ a finite-dimensional graded field extension of $F$. For an arbitrary abelian group $A$-namely for $A=\Gamma_{K} / \Gamma_{F}$ - and a family $a_{1}, a_{2}, \ldots, a_{r}$ of elements of $A$, we say that $a_{1}, a_{2}, \ldots, a_{r}$ are independent if the subgroup $\left\langle a_{1}, a_{2}, \ldots, a_{r}\right\rangle$ of $A$, generated by $a_{1}, a_{2}, \ldots, a_{r}$, equals $\bigoplus_{i=1}^{r}\left\langle a_{i}\right\rangle$. We recall that $K$ is called totally ramified of radical type (TRRT) over $F$ if there are homogeneous elements $t_{1}, \ldots, t_{r} \in F^{*}$ and nonnegative integers $n_{1}, \ldots, n_{r}$ such that the following conditions are satisfied:

(1) $K=F\left[t_{1}^{1 / n_{1}}, \ldots, t_{r}^{1 / n_{r}}\right]$,

(2) $\operatorname{gr}\left(t_{i}^{1 / n_{i}}\right)+\Gamma_{F}(1 \leq i \leq r)$ are independent elements of $\Gamma_{K} / \Gamma_{F}$, with order $n_{i}$, respectively.

One can see that in the same way as for TRRT valued field extensions (see [7, Lemma $4.1]$ ), a totally ramified finite-dimensional graded field extension $K$ of $F$ is TRRT over $F$ if and only if there is a subgroup $G$ of $K^{*} / F^{*}$ such that the mapping $G \rightarrow \Gamma_{K} / \Gamma_{F}$, defined by $x F^{*} \mapsto \operatorname{gr}(x)+\Gamma_{F}$, is a group isomorphism.

Lемма 2.1. Let $F$ be a graded field and $K$ a totally ramified finite-dimensional graded field extension of $F$. Then, $K$ is TRRT over $F$.

Proof. Let $\left(x_{i}\right)_{i=1}^{r}$ be a family of nonzero homogeneous elements of $K$ such that $\left(\operatorname{gr}\left(x_{i}\right)+\right.$ $\left.\Gamma_{F}\right)_{i=1}^{r}$ is a basis of $\Gamma_{K} / \Gamma_{F}$ (i.e., $\left.\Gamma_{K} / \Gamma_{F}=\bigoplus_{i=1}^{r}\left\langle\operatorname{gr}\left(x_{i}\right)+\Gamma_{F}\right\rangle\right)$. Let $n_{i}$ be the order of $\operatorname{gr}\left(x_{i}\right)+$ $\Gamma_{F}(1 \leq i \leq r)$ and set $I=\left\{\bar{m}=\left(m_{1}, \ldots, m_{r}\right) \in \mathbb{N}^{r} \mid 0 \leq m_{i}<n_{i}\right.$ for all $\left.1 \leq i \leq r\right\}$. Let $G$ be the subgroup of $K^{*} / F^{*}$, generated by the elements $x_{i} F^{*}(1 \leq i \leq r)$. For $\bar{m}=\left(m_{1}, \ldots, m_{r}\right) \in$ $I$, let $x^{\bar{m}}=x_{1}^{m_{1}} \cdots x_{r}^{m_{r}}$. Since $K / F$ is totally ramified, then for any $1 \leq i \leq r, x_{i}^{n_{i}} \in F^{*}$ (indeed, we have $K_{0}=F_{0}$ and $\left.\operatorname{gr}\left(x_{i}^{n_{i}}\right) \in \Gamma_{F}\right)$. Clearly, the elements $x^{\bar{m}}(\bar{m} \in I)$ are pairwise distinct. Hence, $G=\left\{x^{\bar{m}} F^{*} \mid \bar{m} \in I\right\}$ and the map $\phi: G \rightarrow \Gamma_{K} / \Gamma_{F}, x^{\bar{m}} F^{*} \mapsto \operatorname{gr}\left(x^{\bar{m}}\right)+\Gamma_{F}$, is a group isomorphism.

Let $F$ be a graded field and $D$ a GCDA over $F$. We recall that in the same way as for valued central division algebras, $D$ is called nicely semiramified (NSR) (over $F$ ) if it has 


\section{Nicely semiramified division algebras over Henselian fields}

unramified and totally ramified radical-type maximal graded subfields. We recall also that $D$ is NSR (over $F$ ) if and only if $\mathrm{Cq}(D)$ is NSR (over Frac $(F)$ ) (see [3, Proposition 6.4]). Moreover, $\mathrm{Cq}(D)$ is NSR (over $\operatorname{Frac}(F)$ ) if and only if $H \mathrm{Cq}(D)$ is NSR (over $H \operatorname{Frac}(F)$ ). Indeed, assume that $\mathrm{Cq}(D)$ is NSR and let $K$ (resp., $L$ ) be an inertial (resp., a TRRT) maximal subfield of $\mathrm{Cq}(D)$. Then, by [8, Theorem 1], $H K\left(=K \otimes_{\operatorname{Frac}(F)} H \operatorname{Frac}(F)\right)$ (resp., $\left.H L\left(=L \otimes_{\mathrm{Frac}(F)} H \operatorname{Frac}(F)\right)\right)$ is an inertial (resp., a TRRT) maximal subfield of $H \mathrm{Cq}(D)$. Conversely, if $H \mathrm{Cq}(D)$ is NSR, then it has an inertial (resp., a TRRT) maximal subfield $K^{\prime}$ (resp., $L^{\prime}$ ). So $G K^{\prime}$ (resp., $G L^{\prime}$ ) is an unramified (resp., a totally ramified) maximal graded subfield of $D\left(\cong_{g} G H C q(D)\right)$. Hence $D$ is NSR. Therefore, by the above, $\mathrm{Cq}(D)$ is NSR.

The following lemma is analogous to [7, Theorem 4.4]. It gives equivalent statements for a GCDA over a graded field to be NSR. In condition (3)(i) of this lemma, the graded field extensions $L^{(i)}$ are said to be linearly disjoint if the graded $\operatorname{ring} L^{(1)} \otimes_{F} \cdots \otimes_{F} L^{(k)}$ is a graded field.

Lemмa 2.2. Let $F$ be a graded field and D a GCDA over F. Then the following statements are equivalent:

(1) D is NSR;

(2) $D$ is split by unramified and totally ramified graded field extensions of $F$;

(3) $D \cong_{g}\left(L^{(1)} / F, \sigma_{1}, t_{1}\right) \otimes_{F} \cdots \otimes_{F}\left(L^{(k)} / F, \sigma_{k}, t_{k}\right)$, where $L^{(i)}, \sigma_{i}$, and $t_{i}$ satisfy the following conditions:

(i) $L^{(i)}$ are linearly disjoint cyclic unramified graded field extensions of $F$ with dimension $\left[L^{(i)}: F\right]=n_{i}$ and with Galois group generated by $\sigma_{i}(1 \leq i \leq k)$,

(ii) $t_{i}$ are nonzero homogeneous elements of $F$ such that $\operatorname{gr}\left(t_{i}^{n / n_{i}}\right)+n \Gamma_{F}$ are independent elements of $\Gamma_{F} / n \Gamma_{F}$, with order $n_{i}(1 \leq i \leq k)$, respectively.

Proof. $(1) \Rightarrow(2)$. Obvious.

$(2) \Rightarrow(3)$. Let $K$ (resp., $K^{\prime}$ ) be an unramified (resp., a totally ramified-hence TRRT) graded field extension of $F$ splitting $D$. It follows from the commutative diagrams
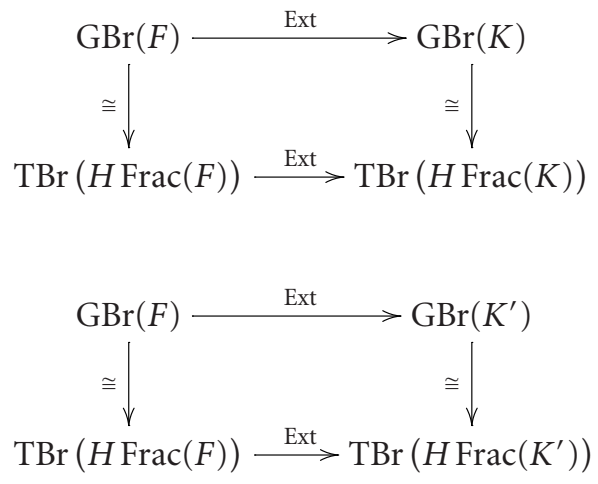

where the horizontal maps are scalar extension homomorphisms and the vertical ones are canonical isomorphisms (see [6, $\operatorname{Remark} 5.10])$, that $H \operatorname{Frac}(K)\left(\operatorname{resp} ., H \operatorname{Frac}\left(K^{\prime}\right)\right.$ $\left.\left(=K^{\prime} \otimes_{\operatorname{Frac}(F)} H \operatorname{Frac}(F)\right)\right)$ is an inertial (resp., a TRRT) field extension of $H \operatorname{Frac}(F)$ splitting $H \mathrm{Cq}(D)$. So, by [7, Theorem 4.4] $H \mathrm{Cq}(D)$ is NSR and we can write $H \mathrm{Cq}(D)=$ $\left(M^{(1)} / H \operatorname{Frac}(F), \tau_{1}, s_{1}\right) \otimes_{H \operatorname{Frac}(F)} \cdots \otimes_{H \operatorname{Frac}(F)}\left(M^{(k)} / H \operatorname{Frac}(F), \tau_{k}, s_{k}\right)$, where $M^{(i)}, \tau_{i}$, and 
$s_{i}$ satisfy the following conditions:

(i) $M^{(i)}$ are linearly disjoint cyclic inertial field extensions of $H \operatorname{Frac}(F)$ with dimension $n_{i}$ and with Galois group generated by $\tau_{i}$, respectively $(1 \leq i \leq k)$,

(ii) $s_{i}(1 \leq i \leq k)$ are elements of $H \operatorname{Frac}(F)^{*}$ such that, if we denote by $v$ the canonical valuation of $H \operatorname{Frac}(F)$, then $\left(n / n_{i}\right) v\left(s_{i}\right)+n \Gamma_{F}$ are independent elements of $\Gamma_{F} / n \Gamma_{F}$, with order $n_{i}$, respectively.

Therefore,

$$
\begin{aligned}
D & \cong_{g} G H \operatorname{Cq}(D) \\
& \cong_{g}\left(G M^{(1)} / G H \operatorname{Frac}(F), \tau_{1}, \widetilde{s}_{1}\right) \otimes_{G H \operatorname{Frac}(F)} \cdots \otimes_{G H \operatorname{Frac}(F)}\left(G M^{(k)} / G H \operatorname{Frac}(F), \tau_{k}, \widetilde{s}_{k}\right) .
\end{aligned}
$$

Indeed, let $D_{i}=\left(M^{(i)} / H \operatorname{Frac}(F), \tau_{i}, s_{i}\right)=\bigoplus_{j=1}^{n_{i}} M^{(i)} x_{i}^{j}$, where $x_{i}^{n_{i}}=s_{i}$ and $x_{i} a=\tau_{i}(a) x_{i}$ for all $a \in M^{(i)}(1 \leq i \leq k)$. Since $M^{(i)} / H \operatorname{Frac}(F)$ is cyclic inertial, then by [5, Remark 3.1] the unramified graded field extension $G M^{(i)} / G H \operatorname{Frac}(F)$ is cyclic and, up to a group isomorphism, $\operatorname{Gal}\left(G M^{(i)} / G H \operatorname{Frac}(F)\right)=\left\langle\tau_{i}\right\rangle$. Moreover, it is clear that for any $d \in M^{(i)}$, we have $\tilde{x}_{i} \tilde{d} \tilde{x}_{i}^{-1}=\widetilde{\tau_{i}(d)}=\tau_{i}(\tilde{d})$. Thus, $G D_{i}=\left(G M^{(i)} / G H \operatorname{Frac}(F), \tau_{i}, \tilde{s}_{i}\right)$. It follows from the canonical isomorphism $\operatorname{TBr}(H \operatorname{Frac}(F)) \cong \operatorname{GBr}(G H \operatorname{Frac}(F))$ that

$$
\begin{aligned}
D( & \left.\cong_{g} G H \mathrm{Cq}(D)\right) \cong_{g} G D_{1} \otimes_{G H \operatorname{Frac}(F)} \cdots \otimes_{G H \operatorname{Frac}(F)} G D_{k} \\
& =\left(G M^{(1)} / G H \operatorname{Frac}(F), \tau_{1}, \widetilde{s}_{1}\right) \otimes_{G H \operatorname{Frac}(F)} \cdots \otimes_{G H \operatorname{Frac}(F)}\left(G M^{(k)} / G H \operatorname{Frac}(F), \tau_{k}, \widetilde{s}_{k}\right) .
\end{aligned}
$$

The conditions of (3) in this lemma are then satisfied.

$(3) \Rightarrow(1)$. By $\left[5\right.$, Theorem 3.11(b)] $\operatorname{Frac}\left(L^{(i)}\right) / \operatorname{Frac}(F)$ are cyclic with $\operatorname{Gal}\left(\operatorname{Frac}\left(L^{(i)}\right) /\right.$ $\operatorname{Frac}(F))=\left\langle\sigma_{i}\right\rangle$. Thus, applying [8, Theorem 1], one can easily see that $H \operatorname{Frac}\left(L^{(i)}\right) /$ $H \operatorname{Frac}(F)$ are cyclic with $\operatorname{Gal}\left(H \operatorname{Frac}\left(L^{(i)}\right) / H \operatorname{Frac}(F)\right) \cong \operatorname{Gal}\left(\operatorname{Frac}\left(L^{(i)}\right) / \operatorname{Frac}(F)\right)$. Moreover, since $\overline{H \operatorname{Frac}\left(L^{(i)}\right)}=L_{0}^{(i)}, \Gamma_{H \operatorname{Frac}\left(L^{(i)}\right)}=\Gamma_{L^{(i)}}, \overline{H \operatorname{Frac}(F)}=F_{0}$, and $\Gamma_{H \operatorname{Frac}(F)}=\Gamma_{F}$, it follows that $H \mathrm{Cq}(D)\left(\cong D \otimes_{F} H \operatorname{Frac}(F) \cong\left(H \operatorname{Frac}\left(L^{(1)}\right) / H \operatorname{Frac}(F), \sigma_{1}, t_{1}\right) \otimes_{H \operatorname{Frac}(F)} \cdots\right.$ $\left.\otimes_{H \operatorname{Frac}(F)}\left(H \operatorname{Frac}\left(L^{(k)}\right) / H \operatorname{Frac}(F), \sigma_{k}, t_{k}\right)\right)$ is NSR (by [7, Theorem 4.4]). So, by the arguments preceding this lemma, $D$ is NSR.

Lemma 2.3. Let $E$ be a Henselian valued field and $D$ a defectless CDA over E. Then the following statements are equivalent:

(1) $D$ is NSR;

(2) GD is NSR.

Proof. $(1) \Rightarrow(2)$. Since $D$ is nicely semiramified-hence tame-over $E$, then $G D$ is a graded central division algebra over GE (see [2, Corollary 4.4(3)]-remark that since $D$ is defectless over $E$, then $[D: E]=[G D: G E]$ ). Let $L$ (resp., $M$ ) be an inertial (resp., a TRRT) maximal subfield of $D$. Then, GL (resp., GM) is an unramified (resp., a totally ramified) maximal graded subfield of $G D$. Hence, $G D$ is nicely semiramified.

$(2) \Rightarrow(1)$. Since $G D$ is nicely semiramified over $G E$, then by Lemma $2.2, G D \cong_{g}\left(L^{(1)} / G E\right.$, $\left.\sigma_{1}, t_{1}\right) \otimes_{G E} \cdots \otimes_{G E}\left(L^{(k)} / G E, \sigma_{k}, t_{k}\right)$, where $L^{(i)} / G E, \sigma_{i}$, and $t_{i}$ satisfy the conditions of 
Lemma 2.2(3). Since $L^{(i)} / G E$ are unramified, then by [5, Theorem 5.2 (see the proof)], there are inertial cyclic field extensions $K^{(i)}$ of $E$ such that-up to a graded isomorphism- $L^{(i)}=G K^{(i)}$ with $\operatorname{Gal}\left(L^{(i)} / G E\right) \cong \operatorname{Gal}\left(K^{(i)} / E\right)$. Let $D^{(i)}=\left(L^{(i)} / G E, \sigma_{i}, t_{i}\right)$. It follows from the canonical isomorphism $\operatorname{TBr}(E) \stackrel{\alpha}{\cong} \operatorname{GBr}(G E)$ that there is a unique-up to an algebra isomorphism-central division algebra $D_{(i)}$ over $E$ such that $D^{(i)} \cong_{g} G D_{(i)}$. By [6, Corollary 5.8], $K^{(i)}$ splits $D_{(i)}$. Hence, up to an isomorphism, $K^{(i)}$ is a maximal subfield of $D_{(i)}$ (see [9, Corollary, page 241]). Write $D_{(i)}=\left(K^{(i)} / E, \sigma_{i}, s_{i}\right)$. Then, $\left(L^{(i)} / G E, \sigma_{i}, t_{i}\right)=$ $D^{(i)} \cong_{g} G D_{(i)}=\left(G K^{(i)} / G E, \sigma_{i}, \tilde{s}_{i}\right) \cong_{g}\left(L^{(i)} / G E, \sigma_{i}, \tilde{s}_{i}\right)$. Accordingly, $\left(L^{(i)} / G E, \sigma_{i}, t_{i}\right) \otimes_{G E}$ $H \operatorname{Frac}(G E) \cong\left(L^{(i)} / G E, \sigma_{i}, \tilde{s}_{i}\right) \otimes_{G E} H \operatorname{Frac}(G E)$ or, equivalently, $\left(H \operatorname{Frac}\left(L^{(i)}\right) / H \operatorname{Frac}(G E)\right.$, $\left.\sigma_{i}, t_{i}\right) \cong\left(H \operatorname{Frac}\left(L^{(i)}\right) / H \operatorname{Frac}(G E), \sigma_{i}, \tilde{s}_{i}\right)$. Therefore, by [9, Lemma, page 278], there is $\alpha_{i} \in$ $H \operatorname{Frac}\left(L^{(i)}\right)$ such that $t_{i}=\left(\prod_{j=1}^{n_{i}} \sigma_{i}^{j}\left(\alpha_{i}\right)\right) \tilde{s}_{i}$. Let $v$ be the canonical valuation of $H \operatorname{Frac}\left(L^{(i)}\right)$. Since $v$ is Henselian, then $\operatorname{gr}\left(t_{i}\right)\left(=v\left(t_{i}\right)\right)=n_{i} v\left(\alpha_{i}\right)+v\left(s_{i}\right)$. So, $\left(n / n_{i}\right) \operatorname{gr}\left(t_{i}\right)+$ $n \Gamma_{E}=\left(n / n_{i}\right) v\left(s_{i}\right)+n \Gamma_{E}\left(\right.$ since $\left.\Gamma_{H \operatorname{Frac}\left(L^{(i)}\right)}=\Gamma_{E}\right)$. We have $[D]=\alpha^{-1}([G D])$, therefore $D \cong$ $\left(K^{(1)} / E, \sigma_{1}, s_{1}\right) \otimes_{E} \cdots \otimes_{E}\left(K^{(k)} / E, \sigma_{k}, s_{k}\right)$. Hence, by [7, Example 4.3], $D$ is NSR.

Theorem 2.4. Let E be a Henselian valued field and D a defectless CDA over E. Then the following statements are equivalent:

(1) D is NSR;

(2) D has inertial and totally ramified maximal subfields;

(3) $D$ is split by inertial and by totally ramified field extensions of $E$.

Proof. $(1) \Rightarrow(2)$. Obvious.

$(2) \Rightarrow(3)$. Obvious.

$(3) \Rightarrow(1)$. Since $D$ is split by an inertial field extension of $E$, then $D$ is tame over $E$ (see [6, page 99]). Hence, GD is a GCDA over GE. Now, let $K$ (resp., $K^{\prime}$ ) be an inertial (resp., a totally ramified) field extension of $E$ splitting $D$. Then $G K$ (resp., $G K^{\prime}$ ) is an unramified (resp., a totally ramified) graded field extension of $G E$ that splits $G D$. So, by Lemmas 2.2 and $2.3, D$ is NSR.

\section{References}

[1] M. Boulagouaz, The graded and tame extensions, Commutative Ring Theory (Fès, 1992), Lecture Notes in Pure and Appl. Math., vol. 153, Marcel Dekker, New York, 1994, pp. 27-40.

[2] L Le gradué d'une algèbre à division valuée [The associated graded ring of a valued division algebra], Comm. Algebra 23 (1995), no. 11, 4275-4300 (French).

[3] - Algèbre à division graduée centrale [Central graded division algebra], Comm. Algebra 26 (1998), no. 9, 2933-2947 (French).

[4] O. Endler, Valuation Theory, Springer, New York, 1972.

[5] Y.-S. Hwang and A. R. Wadsworth, Algebraic extensions of graded and valued fields, Comm. Algebra 27 (1999), no. 2, 821-840.

[6] Correspondences between valued division algebras and graded division algebras, J. Algebra 220 (1999), no. 1, 73-114.

[7] B. Jacob and A. R. Wadsworth, Division algebras over Henselian fields, J. Algebra 128 (1990), no. 1, 126-179.

[8] P. Morandi, The Henselization of a valued division algebra, J. Algebra 122 (1989), no. 1, 232243.

[9] R. S. Pierce, Associative Algebras, Graduate Texts in Mathematics, vol. 88, Springer, New York, 1982. 
[10] V. P. Platonov, The Tannaka-Artin problem, and reduced K-theory, Izv. Akad. Nauk SSSR Ser. Mat. 40 (1976), no. 2, 227-261, 469, translated in Math. USSR Izv. 10 (1976), 211-243.

Karim Mounirh: Département des Mathématiques et d'Informatique, Faculté des Sciences Dhar El Mehraz, Université Sidi Mohamed Ben Abdellah, B.P. 1796, Atlas, Morocco

E-mail address: akamounirh@hotmail.com 


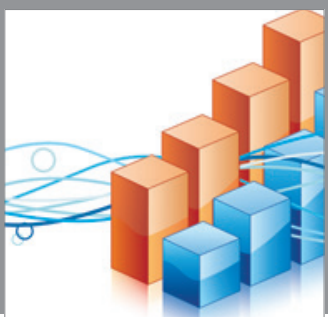

Advances in

Operations Research

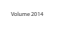

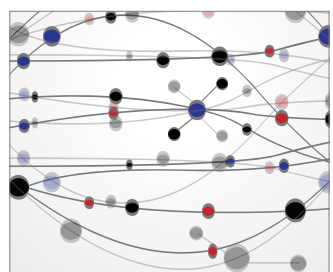

\section{The Scientific} World Journal
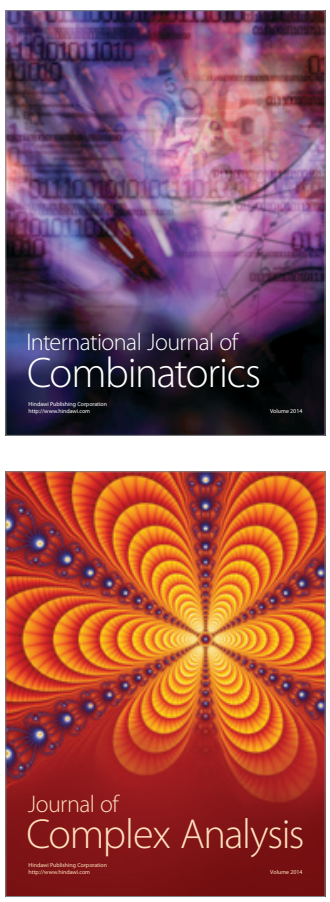

International Journal of

Mathematics and

Mathematical

Sciences
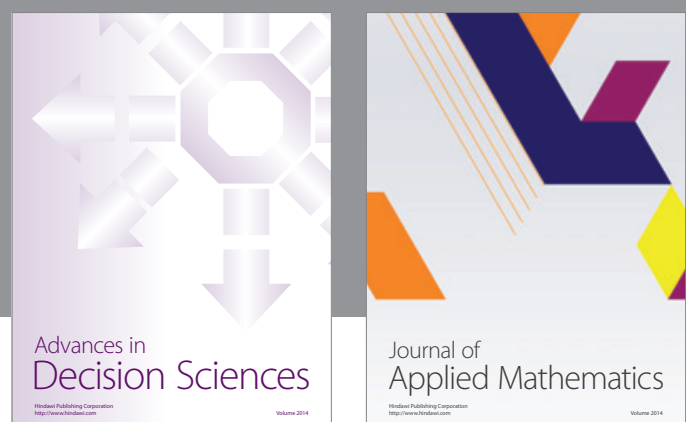

Journal of

Applied Mathematics
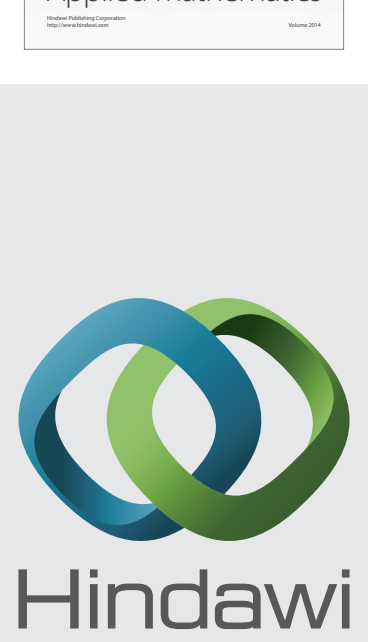

Submit your manuscripts at http://www.hindawi.com
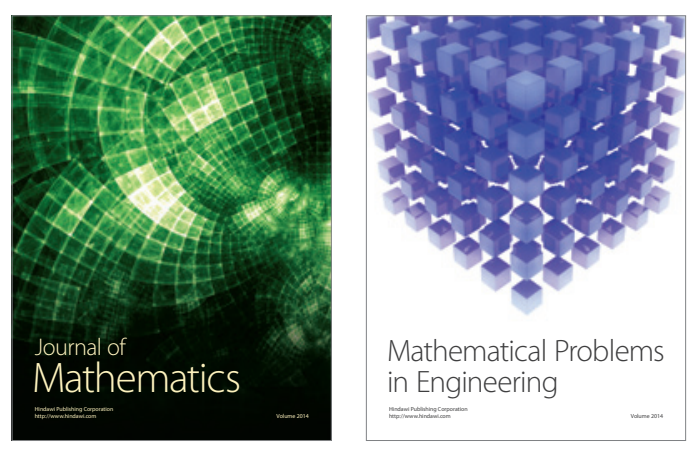

Mathematical Problems in Engineering
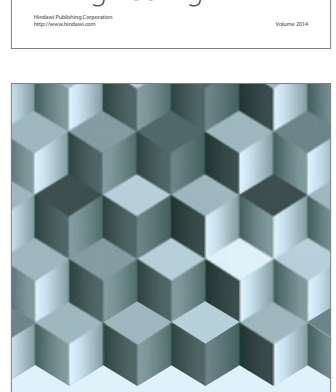

Journal of

Function Spaces
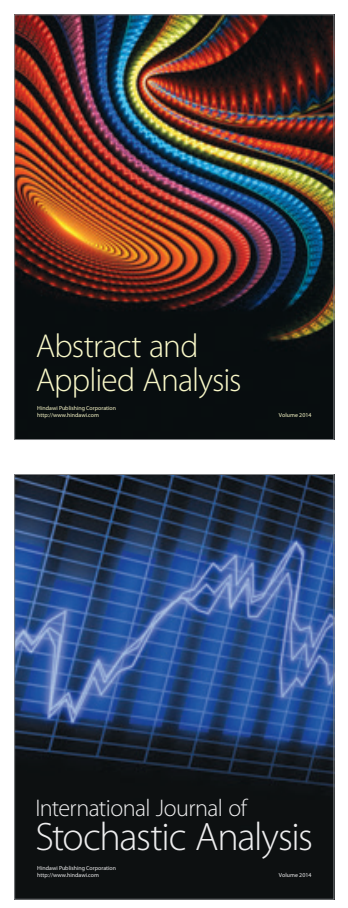

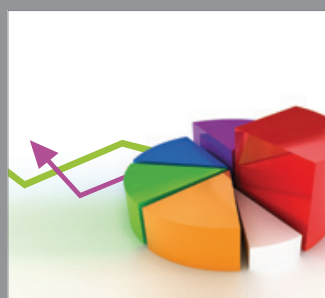

ournal of

Probability and Statistics

Promensencen
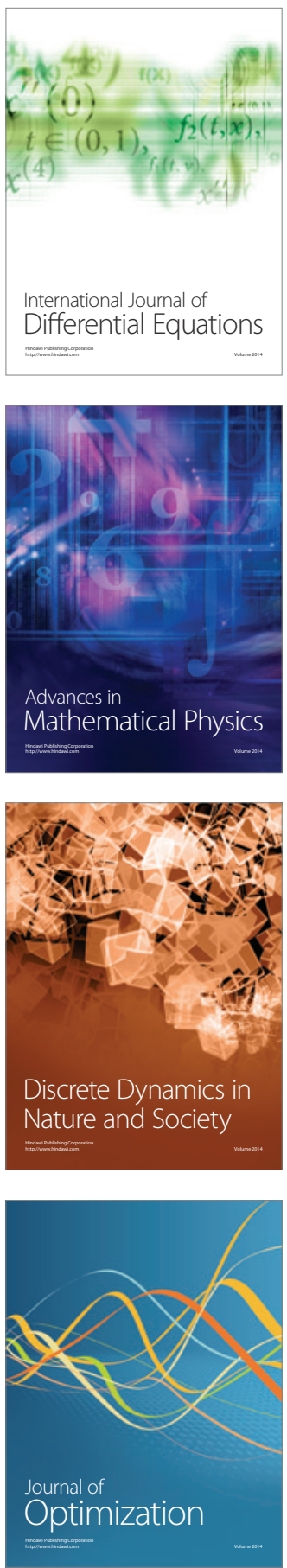